\title{
Restoration of hypothalamic lipid sensing normalizes energy and glucose homeostasis in overfed rats
}

\author{
Alessandro Pocai, ${ }^{1}$ Tony K.T. Lam, ${ }^{1}$ Silvana Obici, ${ }^{1}$ Roger Gutierrez-Juarez, ${ }^{1}$ Evan D. Muse, ${ }^{1}$ \\ Arduino Arduini, ${ }^{2}$ and Luciano Rossetti ${ }^{1}$
}

1Departments of Medicine and Molecular Pharmacology, Diabetes Research Center, Albert Einstein College of Medicine, New York, New York, USA. 2Iperboreal Pharma S.r.l. Via Roma, Pescara, Italy.

\begin{abstract}
Short-term overfeeding blunts the central effects of fatty acids on food intake and glucose production. This acquired defect in nutrient sensing could contribute to the rapid onset of hyperphagia and insulin resistance in this model. Here we examined whether central inhibition of lipid oxidation is sufficient to restore the hypothalamic levels of long-chain fatty acyl-CoAs (LCFA-CoAs) and to normalize food intake and glucose homeostasis in overfed rats. To this end, we targeted the liver isoform of carnitine palmitoyltransferase-1 (encoded by the CPT1A gene) by infusing either a sequence-specific ribozyme against CPT1A or an isoform-selective inhibitor of CPT1A activity in the third cerebral ventricle or in the mediobasal hypothalamus (MBH). Inhibition of CPT1A activity normalized the hypothalamic levels of LCFA-CoAs and markedly inhibited feeding behavior and hepatic glucose fluxes in overfed rats. Thus central inhibition of lipid oxidation is sufficient to restore hypothalamic lipid sensing as well as glucose and energy homeostasis in this model and may be an effective approach to the treatment of diet-induced obesity and insulin resistance.
\end{abstract}

\section{Introduction}

Worldwide prevalence of obesity has been on the rise, along with its dreaded health consequences: diabetes, vascular diseases, and hypertension $(1,2)$. Obesity is due to a mismatch between caloric intake and energy expenditure in susceptible individuals. Redundant molecular and neural mechanisms control energy and glucose homeostasis (3-9). Among them, the integration of nutritional cues within the hypothalamus is emerging as a critical step in the metabolic and behavioral adaptation to changes in energy balance. The hypothalamic metabolism of fatty acids can profoundly modify feeding behavior and glucose metabolism (10-13) and has been proposed to function as a biochemical sensor for nutrient availability that in turn exerts negative feedback on nutrient intake $(13,14)$ and glucose production $(10,15-17)$. In this regard, the hypothalamic levels of long-chain fatty acyl-CoAs (LCFA-CoAs) can be increased by enhanced esterification of circulating or central lipids $(10,16)$ and/or by the local inhibition of lipid oxidation (18). These interventions also result in marked inhibition of feeding behavior and liver glucose fluxes $(10,15,16,18)$ (Figure 1A).

Sprague-Dawley rats presented with a highly palatable (lardsupplemented) diet promptly double their daily caloric intake and develop severe hepatic insulin resistance within just 3 days $(19,20)$. In this model the central administration of the long-chain fatty acid (LCFA) oleic acid fails to decrease food intake and glucose production (21). Is defective sensing of lipids within the hypothalamus partly responsible for the defective behavioral and metabolic adap-

Nonstandard abbreviations used: ACC, acetyl-CoA carboxylase; AMPK, AMPactivated protein kinase; CPT1, carnitine palmitoyltransferase-1; G6Pase, glucose6-phosphatase; LCFA, long-chain fatty acid; LCFA-CoA, long-chain fatty acyl-CoA; $\mathrm{MBH}$, mediobasal hypothalamus; OF, overfed; PF, pair-fed; SC, standard chow.

Conflict of interest: L. Rossetti, A. Arduini, and S. Obici declare that they have a financial interest in the development of inhibitors of fat oxidation as therapeutic agents.

Citation for this article: J. Clin. Invest. 116:1081-1091 (2006). doi:10.1172/JCI26640. tation in this model? Here we postulated that a critical biochemical underpinning for these defects is that an increase in lipid availability fails to increase the hypothalamic levels of LCFA-CoAs. The associated impairment in hypothalamic nutrient sensing in turn contributes to the susceptibility to obesity and insulin resistance in response to voluntary overfeeding. Consistent with this postulate, rescuing the nutritional regulation of hypothalamic LCFA-CoA levels should restore energy and glucose homeostasis by reinstating this central signal of nutrient abundance (Figure 1A).

To address this hypothesis we used molecular and pharmacologic inhibition of the liver isoform of carnitine palmitoyltransferase-1 (CPT1A; Supplemental Figure 1; supplemental material available online with this article; doi:10.1172/JCI26640DS1) as experimental tools designed to increase the hypothalamic levels of LCFA-CoAs in overfed (OF) rats to the levels observed in rats fed a standard chow (SC rats). These experimental manipulations allowed us to examine whether restoring hypothalamic lipid sensing in OF rats is sufficient to curtail food intake and liver glucose fluxes and to restore the responses to an acute increase in lipid availability. Here we show that inhibition of lipid oxidation within the hypothalamus is sufficient to restore lipid sensing and to suppress food intake and hepatic glucose production.

\section{Results}

Short-term overfeeding impairs bypothalamic lipid sensing. We postulated that a defect in hypothalamic lipid sensing accounts at least in part for persistent hyperphagia and for the rapid onset of hepatic insulin resistance following short-term voluntary overfeeding. To examine the impact of a physiological increase in circulating lipids on the mediobasal hypothalamus (MBH) levels of LCFA-CoAs, we placed chronic catheters in rats and then infused a lipid emulsion designed to double plasma LCFAs (Figure 1, B and C). As previously reported, the increase in circulating LCFAs resulted in an approximately 2-fold increase in the MBH levels of LCFA-CoAs in SC rats 
A
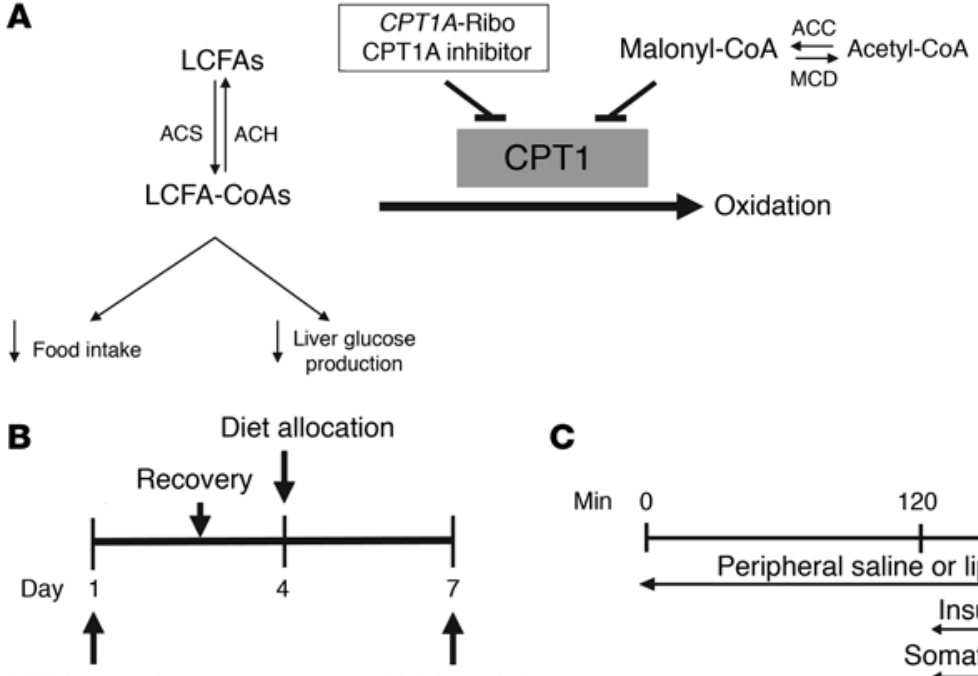

MBH cannulae or catheters

Lipid or oleic acid infusion
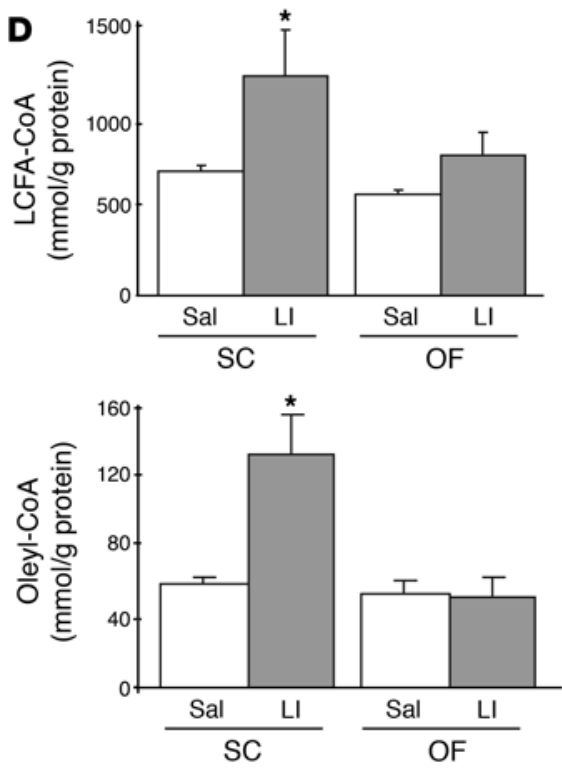

Figure 1

Short-term overfeeding impairs hypothalamic lipid sensing. (A) Selective steps within the hypothalamic metabolism of LCFAs. Administration of systemic and central LCFAs and inhibition of CPT1 increased the cellular concentration of LCFA-CoAs, leading to inhibition of food intake and glucose production. $\mathrm{ACH}$, acyl-CoA hydrolase; ACS, acyl-CoA synthetase; MCD, malonyl-CoA decarboxylase. (B) Experimental protocol. MBH cannulae (for oleic acid infusion) or venous and arterial catheters (for lipid infusion) were implanted on day 1. On day 4 , rats were divided into SC and OF groups. 3 days later the rats were infused with systemic lipid, $\mathrm{MBH}$ oleic acid, or respective vehicle controls (see Methods). (C) Experimental protocol for lipid infusion. (D) Total LCFA-CoA and oleyl-CoA levels in the MBH in SC and OF rats following 4 hours of saline (Sal) or lipid infusion (LI). (E) Oleyl-CoA levels in the $\mathrm{MBH}$ in SC and OF rats following 6 hours of vehicle ( $10 \%$ hydroxypropyl- $\beta$-cyclodextrin) or oleic acid (OA) infusion. ${ }^{*} P<0.05$ versus Sal.
(Figure 1D; lipid versus saline in SC). Conversely, a similar increase in circulating lipids failed to significantly increase LCFA-CoA levels in the $\mathrm{MBH}$ of OF rats (Figure 1D; lipid versus saline in $\mathrm{OF}$ ).

In order to delineate whether this observation was due to defective transfer of LCFAs across the blood-brain barrier or to alterations in cellular fatty acid metabolism, we next infused oleic acid directly within the MBH of SC and OF rats (Figure $1 \mathrm{E}$ ). The $\mathrm{MBH}$ infusion of this LCFA markedly increased the MBH levels of oleyl$\mathrm{CoA}$ in SC rats but failed to increase $\mathrm{MBH}$ oleyl-CoA levels in OF rats. This finding is consistent with (and may account for) the lack of anorectic and metabolic effects of central oleic acid in this model (21). A possible explanation for the muted effect of increased lipid availability on the MBH accumulation of LCFA-CoAs is that the cellular metabolism of esterified LCFA is accelerated in OF compared with SC rats. Indeed the activity of acyl-CoA hydrolase was modestly higher (Supplemental Figure 1A), and CPT1 activity was significantly ( 42\%) and selectively increased, in the MBH (arcuate nuclei) of OF rats (Figure 2A). Hypothalamic nuclei express 2 isoforms of CPT1, the muscle isoform encoded by the CPT1B gene and the liver isoform encoded by the CPT1A gene. The latter is the prevalent isoform in the arcuate (Figure 2A). Short-term overfeeding did not increase the expression of either CPT1 transcript in the arcuate, suggesting that the increased enzymatic activity is likely due to increased protein stability rather than to increased gene expression (Figure 2A). The cellular level of malonyl-CoA, a potent endogenous inhibitor of CPT1, largely determines the in vivo activity of CPT1A. To further examine potential mechanisms responsible for the increased turnover of LCFA-CoAs in the $\mathrm{MBH}$ of OF rats, we next measured the $\mathrm{MBH}$ levels of malonyl-CoA as well as the protein expression of key enzymes involved in its metabolism. Acetyl-CoA carboxylase (ACC) catalyzes the formation of malonyl-CoA from acetyl-CoA (Figure 1A), and its activity is decreased by phosphorylation. ACC phosphorylation (pACC) was markedly increased in the $\mathrm{MBH}$ of $\mathrm{OF}$ compared with that of $\mathrm{SC}$ rats (Figure 2B). Consistent with this finding, the $\mathrm{MBH}$ levels of malonyl-CoA were approximately $70 \%$ lower in OF than in 
A
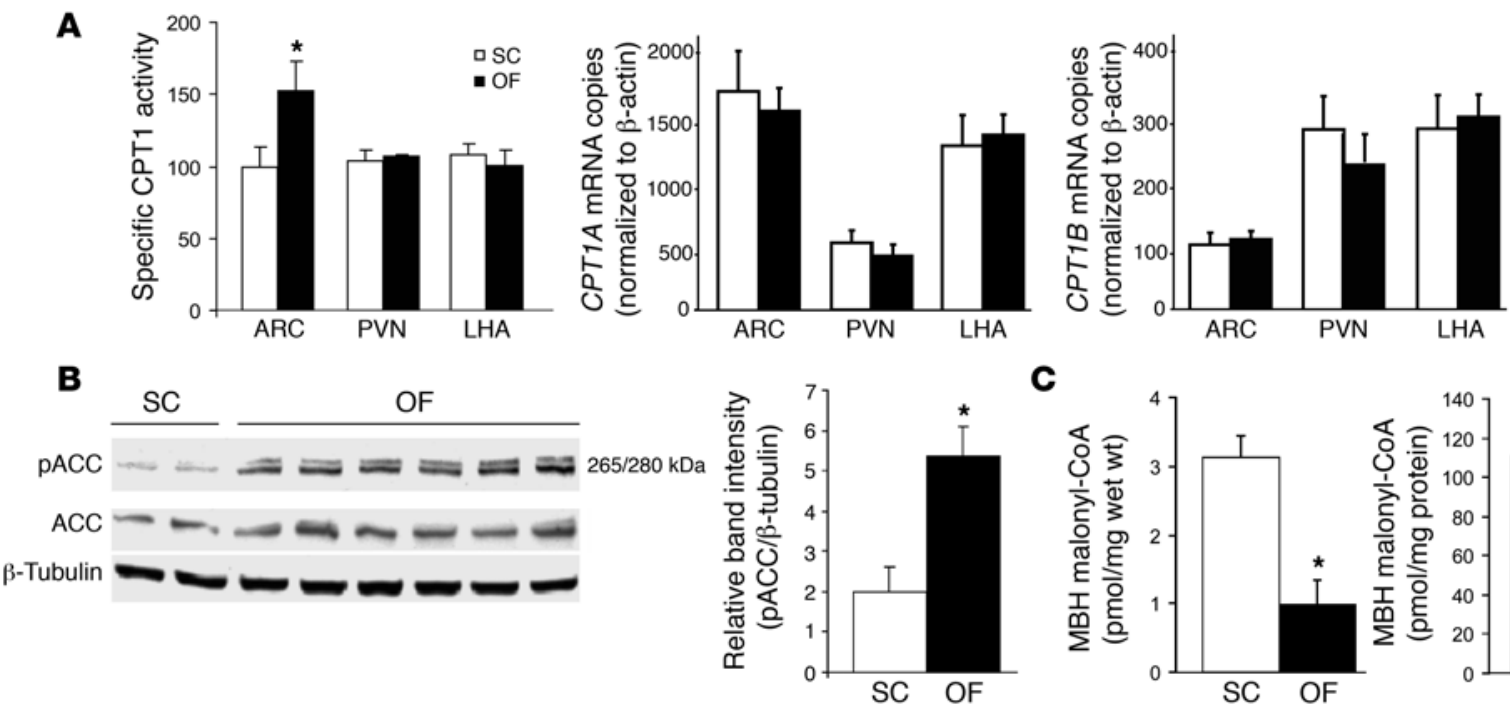

C

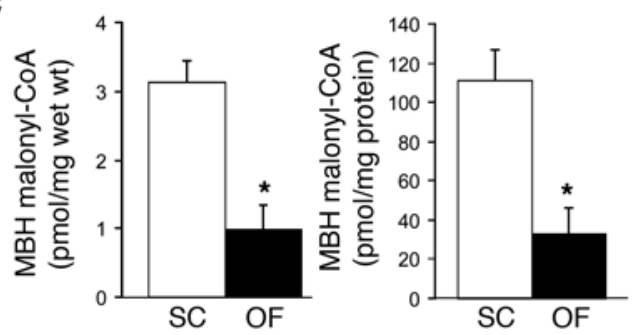

Figure 2

Short-term overfeeding increases the in vivo activity of CPT1 in the arcuate. (A) CPT1-specific activity in the arcuate nucleus (ARC), periventricular nucleus (PVN), and lateral hypothalamic area (LHA) of SC and OF rats. CPT1A and CPT1B mRNA measured in the arcuate nucleus, periventricular nucleus, and lateral hypothalamic area of SC and OF rats. (B) Western blot analysis of MBH-phosphorylated ACC (pACC) and total ACC. Band intensity of pACC was normalized to that of $\beta$-tubulin. Results are expressed as average of 4 different Western blots. (C) MBH malonyl-CoA levels in SC and OF rats expressed as $\mathrm{pmol} / \mathrm{mg}$ wet wt and as $\mathrm{pmol} / \mathrm{mg}$ protein. ${ }^{\star} P<0.05$ versus SC.

$\mathrm{SC}$ rats (Figure $2 \mathrm{C}$ ). On the other hand, we could not detect significant changes in the $\mathrm{MBH}$ expression of AMP-activated protein kinase (AMPK) and fatty acid synthase nor in the phosphorylation of AMPK (Supplemental Figure 2A). Taken together, these observations raise the possibility that decreasing oxidative metabolism of LCFAs within the MBH could restore hypothalamic lipid sensing and curtail feeding behavior in OF rats (Figure 1A).

Inbibition of bypothalamic CPT1A activity decreases food intake in $O F$ rats. Central inhibition of CPT1A activity is sufficient to decrease food intake and body weight in nonobese rats (18). We hypothesized that this anorectic effect is preserved in OF rats and may be sufficient to normalize energy balance. In order to test this hypothesis, we used genetic and pharmacologic approaches designed to curtail the oxidative metabolism of LCFA-CoAs in the $\mathrm{MBH}$ of OF rats (Supplemental Figure 1, B and C, and Figure 3A) in the hope of amplifying the signal generated by an increase in lipid availability (Figure 1A). Thus we first examined whether $\mathrm{MBH}$ inhibition of CPT1A activity decreases food intake following 3 days of voluntary overfeeding in male Sprague-Dawley rats. To this end, we infused SC and OF rats icv with a reversible inhibitor of $\mathrm{CPT} 1 \mathrm{~A}$ activity (Supplemental Figure 1B). As expected the icv administration of the CPT1A inhibitor markedly decreased food intake in SC rats (Figure 3B) (18). Importantly, a similar effect of central CPT1A inhibition was also observed in $\mathrm{OF}$

Table 1 pancreatic insulin clamp rats (Figure 3C). Indeed, the high dose of the CPT1A inhibitor decreased daily caloric intake in OF rats by approximately $40 \%$, to levels $(84 \pm 11 \mathrm{kcal})$ that were similar to those of vehicle-treated $\mathrm{SC}$ rats $(73 \pm 8 \mathrm{kcal}$; Supplemental Figure 3 , A and C).

Inhibition of hypothalamic CPT1A expression decreases food intake in $O F$ rats. We next examined whether silencing the expression of the CPT1A gene (Figure 3, D and E, and Supplemental Figure 3) can reproduce the anorectic effects elicited by the pharmacologic inhibition of hypothalamic CPT1A activity. To this end we injected icv a plasmid containing a ribozyme that specifically cleaves $C P T 1 A$ mRNA (CPT1A-Ribo; Supplemental Figure 1C). Treatment with this ribozyme selectively decreases the expression of CPT1A, but does not alter the expression of CPT1B and that of numerous

Pharmacologic inhibition of hypothalamic CPT1A: general characteristics before and during

$\begin{array}{lcccccc} & \text { SC control } & \text { SC CPT1Ai } & \text { PF control } & \text { PF CPT1Ai } & \text { OF control } & \text { OF CPT1Ai } \\ n & 9 & 10 & 5 & 5 & 11 & 11 \\ \text { Basal } & & & & & & \\ \quad \text { Body weight (g) } & 306 \pm 1 & 301 \pm 6 & 300 \pm 8 & 304 \pm 3 & 311 \pm 5 & 317 \pm 9 \\ \quad \text { Food intake (kcal) } & 73 \pm 3 & 74 \pm 3 & 77 & 77 & 77 & 77 \\ \quad \text { Glucose (mM) } & 8.2 \pm 0.9 & 8.4 \pm 0.2 & 8.2 \pm 0.4 & 8.3 \pm 0.1 & 8.2 \pm 0.5 & 8.3 \pm 0.3 \\ \text { Insulin (ng/ml) } & 1.2 \pm 0.3 & 1.1 \pm 0.2 & 1.2 \pm 0.1 & 0.9 \pm 0.3 & 1.4 \pm 0.2 & 1.3 \pm 0.3 \\ \text { Leptin (ng/ml) } & 0.9 \pm 0.2 & 0.9 \pm 0.1 & 0.8 \pm 0.0 & 1.1 \pm 0.1 & 1.3 \pm 0.3 & 1.4 \pm 0.2 \\ \quad \text { FFA (mM) } & 0.6 \pm 0.1 & 0.5 \pm 0.2 & 0.6 \pm 0.1 & 0.7 \pm 0.2 & 0.8 \pm 0.1 & 0.7 \pm 0.1 \\ \text { Clamp } & & & & & & \\ \quad \text { Glucose (mM) } & 7.8 \pm 0.3 & 8.1 \pm 1.1 & 7.7 \pm 0.2 & 7.8 \pm 0.2 & 7.9 \pm 0.4 & 7.9 \pm 0.2 \\ \text { Insulin (ng/ml) } & 0.8 \pm 0.1 & 0.9 \pm 0.1 & 0.9 \pm 0.1 & 0.9 \pm 0.1 & 0.9 \pm 0.1 & 0.9 \pm 0.1 \\ \text { FFA (mM) } & 0.5 \pm 0.1 & 0.6 \pm 0.1 & 0.5 \pm 0.1 & 0.6 \pm 0.1 & 0.6 \pm 0.0 & 0.7 \pm 0.1\end{array}$

Data are means \pm SEM. The values during clamp represent steady-state levels. Food intake and body weight were measured at the beginning of the clamp. FFA, free fatty acids; HF-CR, calorie intake matched to the group receiving SC with high fat diet; CPT1Ai, CPT1A inhibitor. ${ }^{A} P<0.05$ versus $S C$ and PF. 

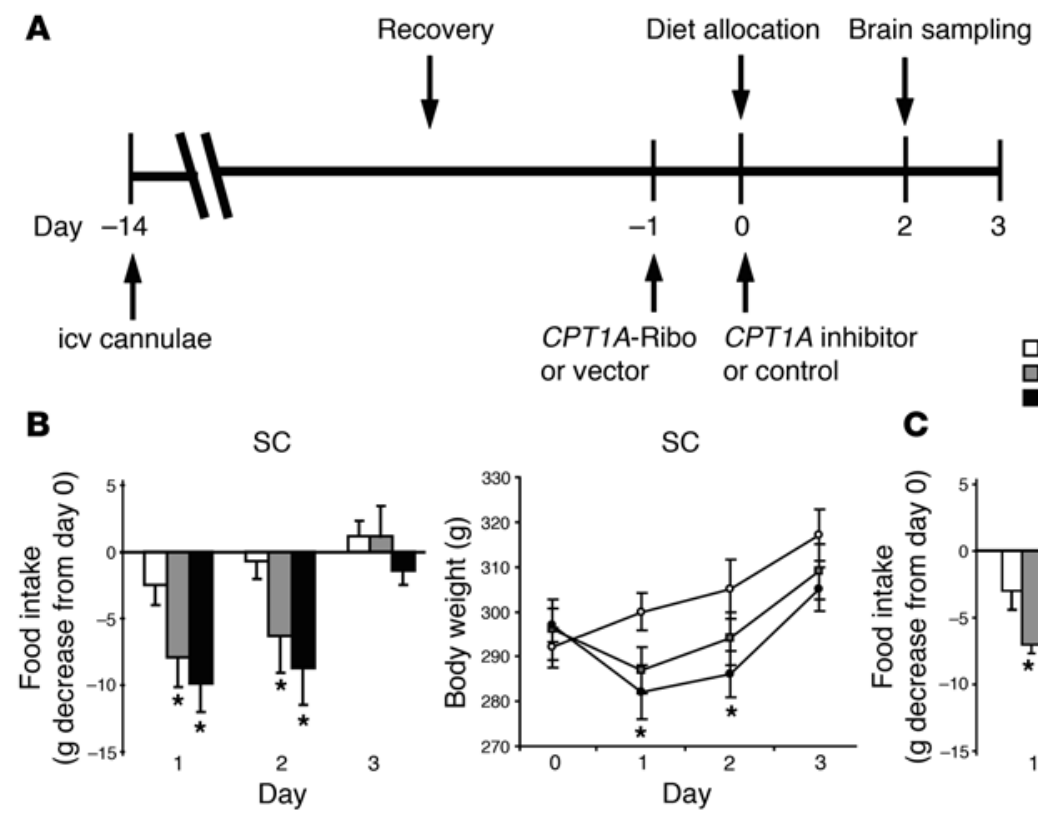

C

Contro 口CPT1A inhibitor (5 pmol) -CPT1A inhibitor ( 25 pmol)
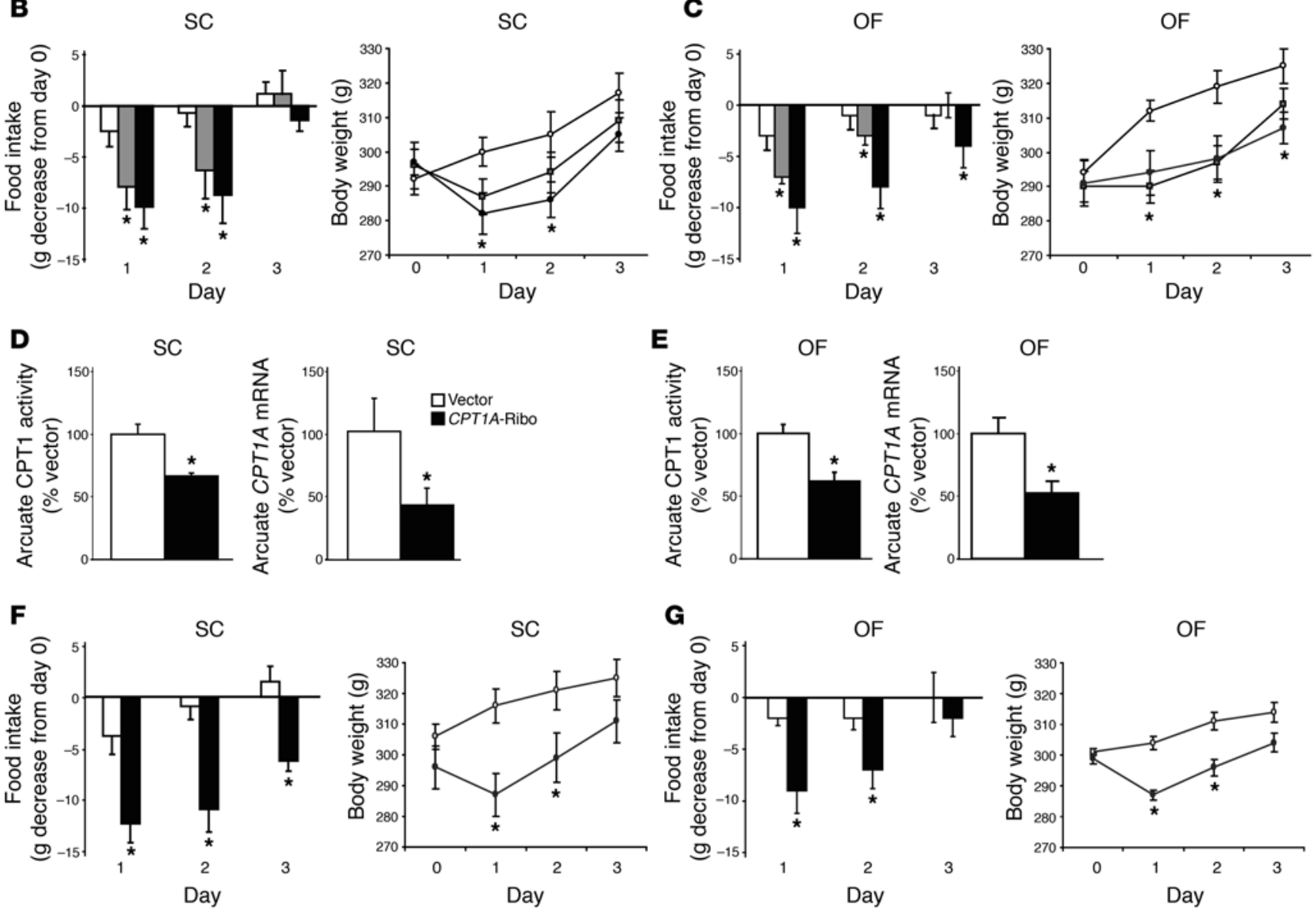

Figure 3

Inhibition of hypothalamic CPT1A activity or expression decreases food intake. (A) Schematic representation of the feeding experiments. (B and C) Daily changes in food intake and body weight induced by icv administration of 2 doses of the CPT1A inhibitor or of its inactive stereoisomer in SC and OF rats. (D and E) Arcuate CPT1 activity and CPT1A expression in arcuate nucleus of SC and OF rats treated with CPT1A-Ribo or vector. (F and $\mathbf{G})$ Changes in food intake and body weight induced by icv administration of $C P T 1 A$-Ribo or vector in $S C$ and $O F$ rats. ${ }^{\star} P<0.05$ versus control and day 0 .

other transcripts and proteins (18). Rats were divided into SC and OF groups and fed the corresponding diets for 2 days (Figure 3A). Prior to icv injections, OF rats markedly increased their daily caloric intake compared with SC rats (Table 1). On the third day, each group received a single icv injection of CPT1A-Ribo or a control vector, and daily food intake was monitored for 72 hours. Injection of the CPT1A-Ribo icv significantly decreased $C P T 1 A$ expression and total CPT1 activity in the arcuate nucleus of both SC and OF rats (Figure 3, D and E). CPT1A-Ribo treatment markedly decreased food intake and body weight gain in SC (Figure 3F) as well as in OF rats (Figure 3G), while their respective control vectors had no discernible effects on these parameters. The anorectic effect of a single injection of CPT1A-Ribo was sustained for at least 2 days (Figure 3, $F$ and $G$ ). As observed with the pharmacologic inhibition, normalizing CPT1 activity within the MBH via transient silencing of CPT1A expression was sufficient to decrease the daily caloric intake of OF rats to the level of SC rats (Supplemental Figure 3). Thus, although the central administration of LCFA failed to decrease food intake in this model (21), inhibition of lipid oxidation within the $\mathrm{MBH}$ is sufficient to restore energy balance in OF rats. 
A

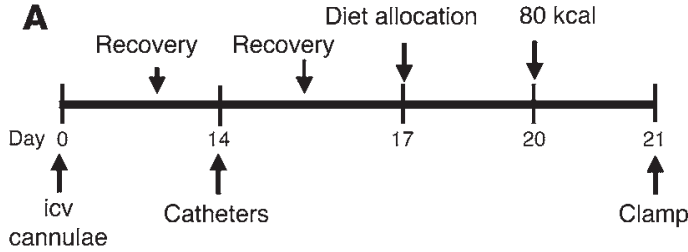

B
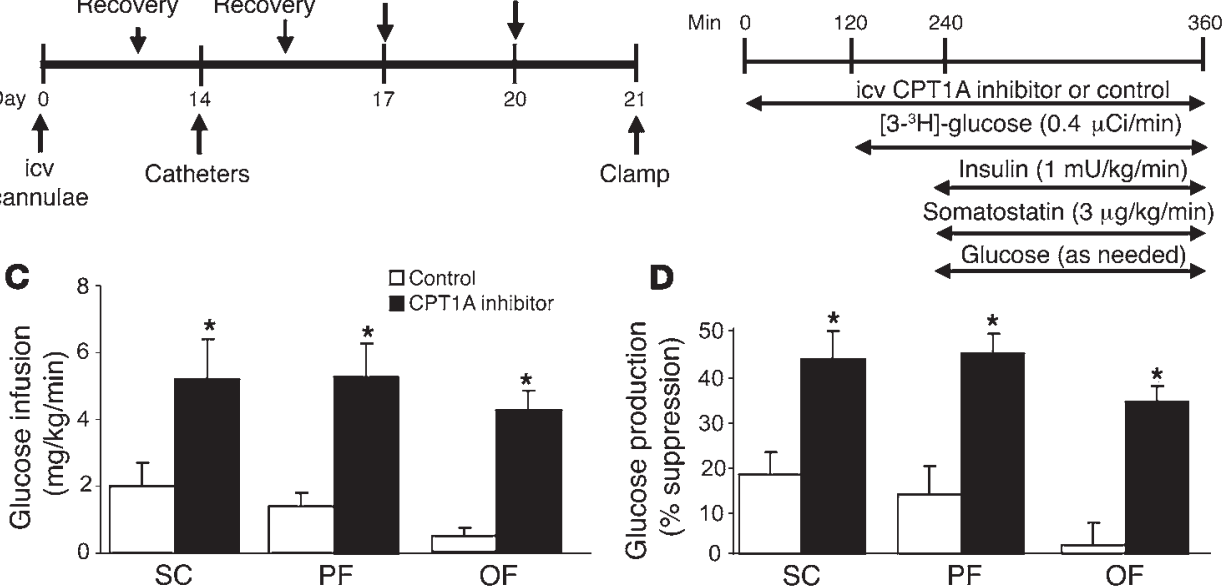

E

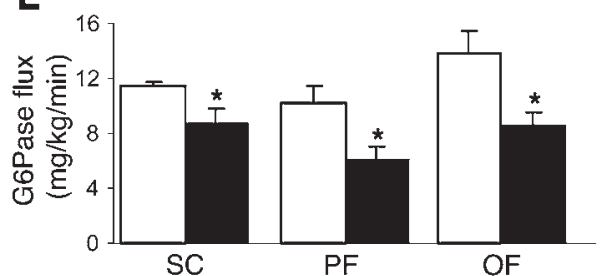

G

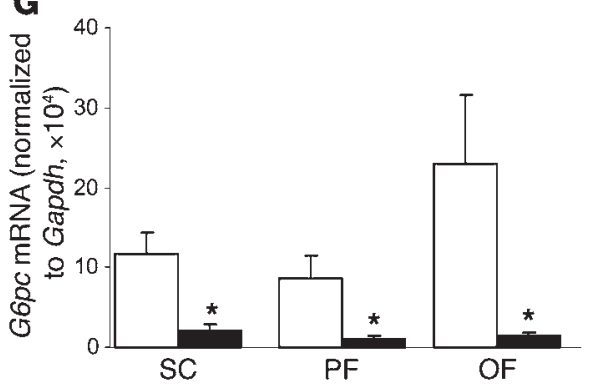

D
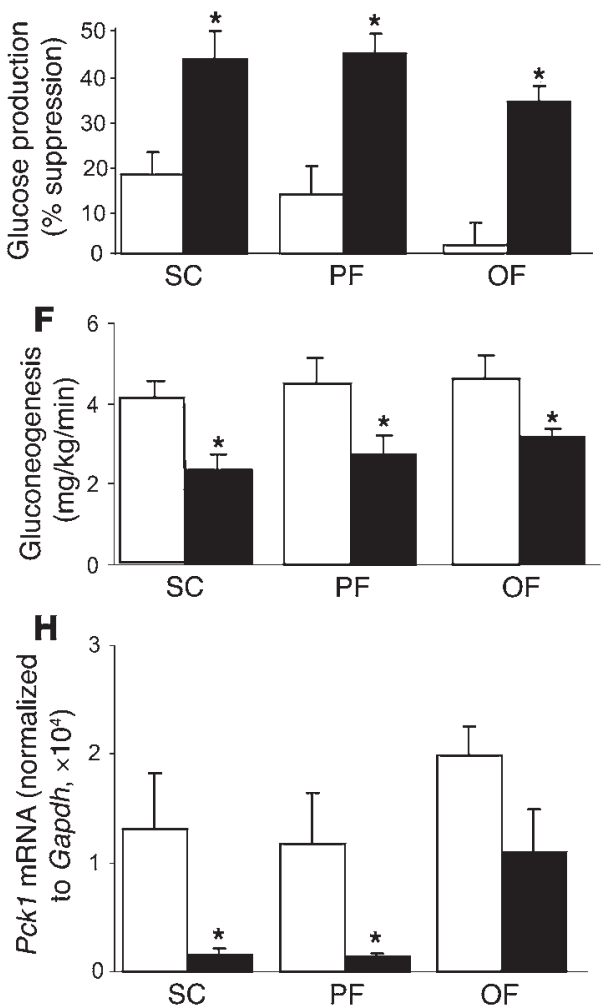

\section{Figure 4}

Inhibition of hypothalamic CPT1A activity restrains liver glucose fluxes in OF rats. Experimental protocol (A). Placement of icv cannulae occurred on day 0 ( 3 weeks before the in vivo study), and venous and arterial catheters were placed on day 14 . Following recovery from the surgery (day $17)$, rats were divided in 3 groups: $\mathrm{SC}, \mathrm{OF}$, and PF. On the evening prior to the pancreatic-insulin clamp, all rats received a fixed portion of calories $(80 \mathrm{kcal})$ to ensure a comparable post-absorptive state at the start of the metabolic experiments. (B) Pancreatic-insulin clamp. Rats received icv infusion of the pharmacologic inhibitor of CPT1A or its inactive stereoisomer for 6 hours before and during the clamp (see Methods). (C) Glucose infusion rate during the clamp in SC, PF, and OF rats. (D) Suppression of glucose production during the clamp period express as percent decrease from basal glucose production. Central inhibition of fatty acid oxidation markedly suppressed G6Pase flux (E), gluconeogenesis (F), and the expression of key genes involved in glucose metabolism, G6Pc (G) and Pck1 (H). ${ }^{\star} P<0.05$ versus control.
Potential mechanisms of hyperphagia in OF rats. Among the downstream $\mathrm{MBH}$ targets of the main anorexigenic hormone leptin are the serine/threonine kinase AMPK (14) and the suppressor of cytokine signaling-3 (SOCS3) (22). Leptin decreases the phosphorylation and activity of AMPK in the arcuate, and this effect appears to be required for its anorexigenic effect (14). Short-term overfeeding failed to alter the expression and phosphorylation of AMPK in the MBH (Supplemental Figure 2A). SOCS3 is a potent inhibitor of leptin signaling and is emerging as an important determinant of leptin resistance in models of chronic obesity (23). However, we did not observe an increase in SOCS3 expression in the $\mathrm{MBH}$ of OF rats (Supplemental Figure 2B). To gain insight into potential mechanisms responsible for the changes described above in the feeding behavior in OF rats, we next analyzed the effect of overfeeding and central CPT1A inhibition on the gene expression of the orexigenic neuropeptides neuropeptide Y (NPY) and agouti-related peptide (AGRP). OF rats showed a significant increase in AGRP and NPY mRNA. Importantly, these increases were negated by the icv infusion of the CPT1A inhibitor (Supplemental Figure 3B). Importantly, central inhibition of CPT1 did not display any effect on conditioned taste aversion Supplemental Figure 2C).
Inbibition of bypothalamic CPT1A activity decreases glucose production in OF rats. Insulin and LCFAs act in the MBH to inhibit liver glucose production $(10,16,18,24)$. However, short-term overfeeding leads to impairment of hepatic insulin action (20) as well as resistance to the effects of LCFAs on liver glucose fluxes $(16,21)$. Since in SC rats the central inhibition of CPT1A activity restrains liver glucose fluxes independently of CPT1A's effects on energy balance (18), we next examined whether this intervention can reverse the hepatic insulin resistance induced by short-term overfeeding. To this end we combined the icv infusion of the CPT1A inhibitor or its inactive stereoisomer (control) with pancreatic insulin clamp studies designed to maintain plasma insulin concentration at basal levels (Figure 4, A and $B$ ). To discern the relative contributions of the increased caloric intake versus the macronutrient composition of the test diets, we also included a group of rats receiving a fixed daily amount of the high-fat diet designed to match the daily caloric consumption of the SC rats (pair-fed [PF] group; Table 1). When the inactive stereoisomer was infused icv, marginal rates of glucose infusion were required to maintain euglycemia in the 3 experimental groups (SC, $1.9 \pm 0.7 \mathrm{mg} / \mathrm{kg} / \mathrm{min} ; \mathrm{PF}, 1.7 \pm 0.3 \mathrm{mg} / \mathrm{kg} / \mathrm{min}$; and OF, $0.4 \pm 0.2$ $\mathrm{mg} / \mathrm{kg} / \mathrm{min}$ ). On the other hand, when the CPT1A inhibitor was infused icv, the rate of glucose infusion required to maintain eug- 

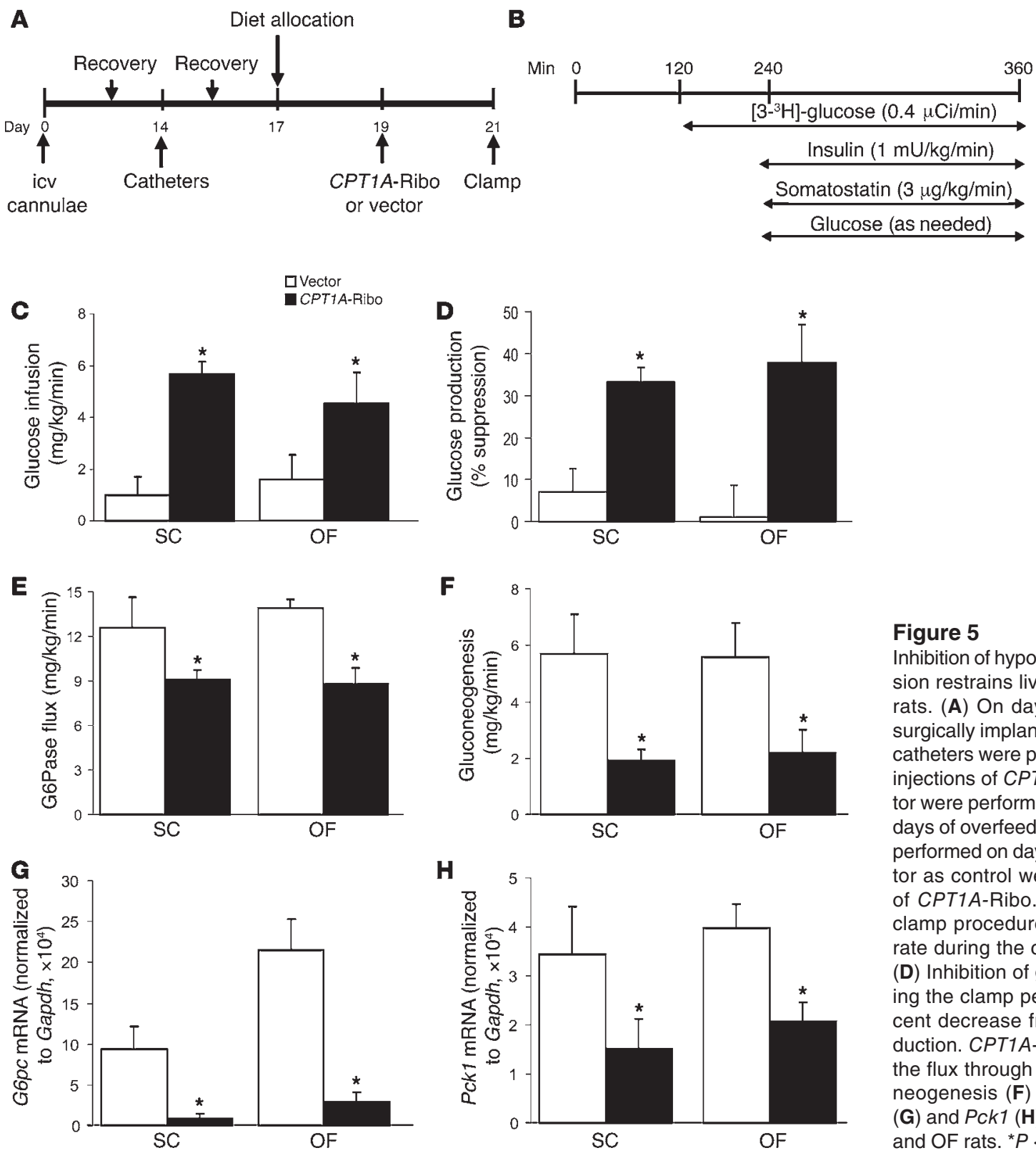

Figure 5

Inhibition of hypothalamic CPT1A expression restrains liver glucose fluxes in $\mathrm{OF}$ rats. (A) On day 0 , icv cannulae were surgically implanted. Arterial and venous catheters were placed on day 14 , and icv injections of CPT1A-Ribo or control vector were performed on day 19 following 2 days of overfeeding. Clamp studies were performed on day 21 . Rats receiving vector as control were pair-fed to the level of CPT1A-Ribo. (B) Pancreatic insulin clamp procedure. (C) Glucose infusion rate during the clamp in SC or OF rats. (D) Inhibition of glucose production during the clamp period expressed as percent decrease from basal glucose production. CPT1A-Ribo markedly reduced the flux through G6Pase (E) and gluconeogenesis $(\mathbf{F})$ and suppressed $G 6 p c$ (G) and Pck1 (H) gene expression in SC and OF rats. ${ }^{*} P<0.05$ versus control. lycemia was markedly and similarly increased in all groups (SC, $4.7 \pm 1.1 \mathrm{mg} / \mathrm{kg} / \mathrm{min} ; \mathrm{PF}, 4.8 \pm 0.8 \mathrm{mg} / \mathrm{kg} / \mathrm{min}$; and OF, $4.2 \pm 0.4$ $\mathrm{mg} / \mathrm{kg} / \mathrm{min}$; Figure $4 \mathrm{C}$ ). Infusion of the CPT1A inhibitor icv failed to significantly alter the levels of glucoregulatory hormones (Table 1) and the rate of glucose uptake (Supplemental Figure 4). On the other hand, this intervention markedly suppressed liver glucose production (Figure 4D and Supplemental Figure 4), and this effect entirely accounted for the effect of central inhibition of fat oxidation on glucose metabolism in all groups.

Glucose production represents the net contribution of gluconeogenesis and glycogenolysis. However, a portion of glucose entering the liver via phosphorylation is also a substrate for dephosphorylation via glucose-6-phosphatase (G6Pase, encoded in rodents by the G6pc gene), creating a futile (glucose) cycle. To delineate the mechanisms by which central inhibition of lipid oxidation modulates liver glucose homeostasis, we estimated the in vivo flux through G6Pase and the relative contribution of glucose cycling, gluconeogenesis, and glycogenolysis to glucose output (Figure 4, E and F, Supplemental Figure 4, and Supplemental Table 1). Central CPT1A inhibition markedly and similarly decreased glucose cycling (Supplemental Figure 4) and the flux through G6Pase (Figure 4E) in parallel to its effects on glucose production (Supplemental Figure 4) in SC, PF, and OF rats. The decrease in glucose production was accounted for by a marked inhibition of gluconeogenesis (Figure 4F) as well as by a more modest decrease in the rate of glycogenolysis (Supplemental Figure 4).

We next assessed the effect of central inhibition of fat oxidation on the expression of G6pc and phosphoenolpyruvate carboxykinase, encoded by the Pck1 gene. Real-time PCR analyses revealed that icv administration of a CPT1A inhibitor markedly decreased liver G6pc and Pck1 mRNA levels (Figure 4, G and H). Thus the decreases in the in vivo flux through G6Pase and gluconeogen- 


\section{Table 2}

Molecular inhibition of hypothalamic CPT1A: general characteristics before and during pancreatic insulin clamp

$\begin{array}{lcccc} & \begin{array}{c}\text { SC pair-fed } \\ \text { vector }\end{array} & \begin{array}{c}\text { SC } \\ \text { CPT1A-Ribo }\end{array} & \begin{array}{c}\text { OF pair-fed } \\ \text { vector }\end{array} & \begin{array}{c}\text { OF } \\ \text { CPT1A-Ribo }\end{array} \\ & 4 & 5 & 6 & 6 \\ \text { Basal } & & & & \\ \quad \text { Body weight (g) } & 293 \pm 7 & 289 \pm 8 & 307 \pm 6 & 299 \pm 5 \\ \quad \text { Food intake (kcal) } & 54 & 56 \pm 3 & 85 & 87 \pm 5 \\ \quad \text { Glucose (mM) } & 8.1 \pm 0.2 & 8.2 \pm 0.3 & 8.2 \pm 0.5 & 8.3 \pm 0.3 \\ \quad \text { Insulin (ng/ml) } & 1.2 \pm 0.6 & 1.1 \pm 0.2 & 1.4 \pm 0.4 & 1.3 \pm 0.3 \\ \text { Leptin (ng/ml) } & 1.3 \pm 0.2 & 0.9 \pm 0.2 & 1.4 \pm 0.4 & 1.3 \pm 0.3 \\ \quad \text { FFA (mM) } & 0.6 \pm 0.2 & 0.6 \pm 0.1 & 0.7 \pm 0.1 & 0.6 \pm 0.2 \\ \text { Clamp } & & & & \\ \quad \text { Glucose (mM) } & 7.6 \pm 0.3 & 7.8 \pm 0.2 & 7.5 \pm 0.4 & 8.2 \pm 0.5 \\ \text { Insulin (ng/ml) } & 0.5 \pm 0.1 & 0.6 \pm 0.1 & 0.5 \pm 0.1 & 0.7 \pm 0.1\end{array}$

Data are means \pm SEM. The values during clamp represent steady-state levels. In this experiment, the food intake in the vector group was matched to that of CPT1A-Ribo group. Food intake and body weight were measured at the begin of the clamp. FFA, free fatty acids.

esis are at least partly due to decreased hepatic expression of these 2 glucoregulatory enzymes.

Inbibition of hypothalamic CPT1A expression decreases glucose production in OF rats. CPT1A-Ribo or vector was injected in SC and OF rats 2 days before the clamp procedure (Figure 5, A and B). Animals receiving the vector injection were pair-fed with the CPT1A-Ribo group in order to match their daily food intake (Table 2). The central administration of CPT1A-Ribo markedly increased the rate of glucose infusion required to maintain euglycemia in $\mathrm{OF}$ as well as $\mathrm{SC}$ rats (Figure 5C). These metabolic effects were independent of changes in caloric intake, since comparisons were only made between pair-fed groups. To investigate the mechanism responsible for the improvement in whole-body glucose homeostasis, we assessed glucose kinetics using a tracer dilution technique. The levels of glucoregulatory hormones (Table 2) and the rate of glucose uptake (Supplemental Figure 5) were not significantly affected by diet and/or CPT1A-Ribo. The improvement in whole-body insulin sensitivity observed following central decrease in CPT1A expression was entirely accounted for by decreased glucose production (Figure 5D and Supplemental Figure 5). We further delineated the mechanisms by which CPT1A-Ribo inhibits liver glucose output. Supplemental Table 2 displays the specific activities of liver and plasma metabolites used to calculate the contribution of lactatederived phosphoenolpyruvate and plasma glucose to the hepatic glucose-6-phosphate pool. Molecular inhibition of CPT1A markedly decreased glucose cycling (Supplemental Figure 5) and the flux through G6Pase (Figure 5E) in SC and OF rats. Central inhibition of fat oxidation also led to marked suppression of the rate of gluconeogenesis (Figure 5F), while glycogenolysis was not consistently altered (Supplemental Figure 5). The marked suppression of liver glucose fluxes was associated with a robust suppression of the hepatic mRNA levels of G6pc and Pck1 (Figure 5, G and H). Thus decreased hypothalamic expression of CPT1A decreased liver glucose fluxes to a similar degree in SC and OF rats, at least in part by decreasing the expression of Pck1 and G6pc.

$M B H$ infusion of a CPT1A inhibitor restores central lipid sensing in $O F$ rats. The effects of the central inhibition of CPT1A activity and/ or expression on food intake and liver glucose homeostasis sug- gest that this intervention might have restored the hypothalamic sensing of lipids in OF rats. To directly test this hypothesis, OF rats received $\mathrm{MBH}$ infusions of either a CPT1A inhibitor or its inactive stereoisomer while the availability of circulating lipids was experimentally enhanced by lipid infusion. We then assessed the effects of these procedures on the $\mathrm{MBH}$ levels of LCFA-CoAs and on glucose homeostasis. To this end we used a combination of bilateral $\mathrm{MBH}$ infusion, systemic lipid infusion, and pancreatic basal insulin clamp in conscious OF rats (Figure 6A). This protocol generated stable and similar levels of glucoregulatory hormones and free fatty acids in all groups. Under these experimental conditions, hyperlipidemia induced hyperglycemia in OF rats (Figure 6A) due to an approximately $20 \%$ increase in the rate of glucose production. Importantly, the inhibition of $\mathrm{MBH}$ CPT1A activity was sufficient to prevent hyperglycemia in OF rats, and this effect was entirely accounted for by a marked decrease in glucose production (Figure 6A). These results suggest that the inhibition of $\mathrm{MBH}$ lipid oxidation restores lipid sensing, perhaps by increasing the cellular levels of LCFA-CoAs. As shown in Figure 6B, lipid infusion increased MBH LCFA-CoA levels in OF rats - only in the presence of $\mathrm{MBH}$ CPT1A inhibition - to a degree similar to that achieved with lipid infusion alone in the SC rats (Figure 1B). Our results indicate that following short-term overfeeding, an increase in lipid availability fails to increase the levels of LCFACoAs within the MBH. Restoring MBH LCFA-CoA levels by inhibition of CPT1A activity appears to be sufficient to improve energy balance and glucose homeostasis (Figure 6C).

\section{Discussion}

Type 2 diabetes and obesity are classical examples of diseases caused by gene/environment interactions (25). Secular changes in our lifestyle that include increases in caloric intake and longer time spent performing sedentary activities precipitate their phenotypic manifestations by acting on a genetic/familiar predisposition $(26,27)$. The hypothalamus is emerging as a critical site for the integration of nutritional, endocrine, and neural cues signaling the body's metabolic and nutritional status. These signals should normally activate a negative feedback loop between the availability of nutrients and their intake and metabolism $(10,13,15,16,18$, $24,28,29)$. It is therefore logical to postulate that a rapid onset of hypothalamic resistance to multiple signals, such as leptin, insulin, and fatty acids, could contribute to the susceptibility to weight gain and insulin resistance in predisposed individuals and animals. In this regard, central resistance to the behavioral and metabolic effects of each of these circulating factors has been reported in various animal models of diet-induced obesity and insulin resistance (30-33). Taken together, these observations raise the possibility that the disruption of a critical component within this regulatory loop confers susceptibility to diet-induced obesity and insulin resistance. Indeed, acquired defects within this regulatory loop have been described in various rodent models of established diet-induced obesity. These experiments have largely focused on the development of leptin resistance that is due partly to defective transport of the hormone across the blood-brain barrier $(30,32)$ and partly to defective leptin signaling, particularly in the arcuate nuclei of the hypothalamus $(23,30,31)$. 


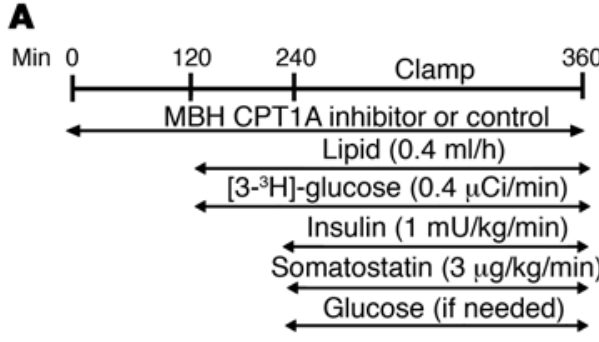

B

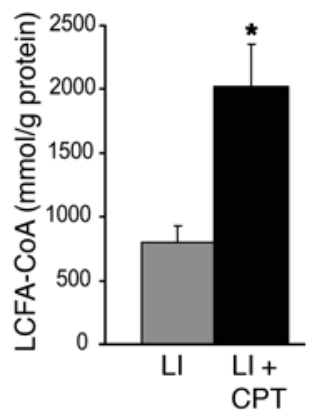

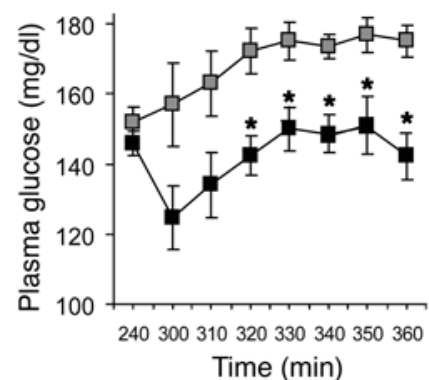

Time (min)

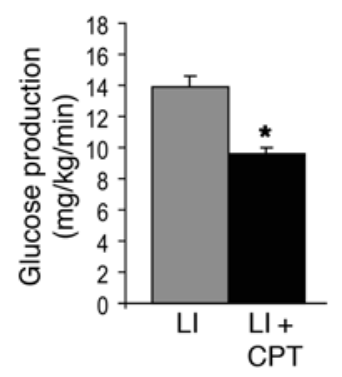

C

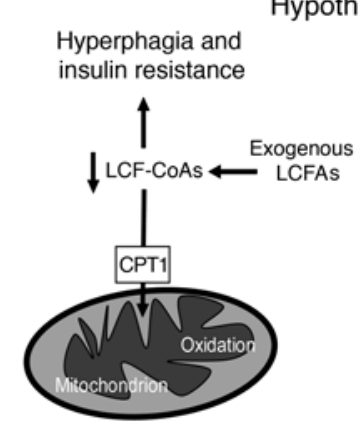

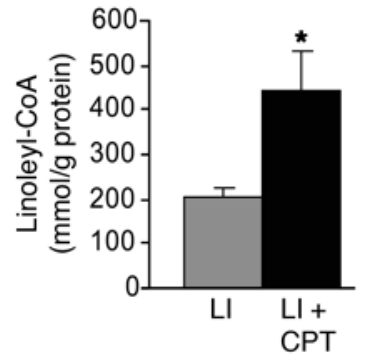

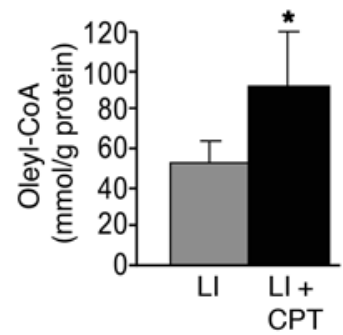

\section{Figure 6}

Hypothalamic inhibition of lipid oxidation restores central lipid sensing in OF rats. (A) Experimental protocol. Male Sprague-Dawley rats fed a high-fat diet for 3 days were infused for in the $\mathrm{MBH}$ for 6 hours. After 2 hours rats received intravenous saline or a lipid emulsion and [3-3 $\mathrm{H}$-glucose for 4 hours, and a pancreatic basal insulin clamp $(1 \mathrm{mU} / \mathrm{kg} / \mathrm{min})$ was performed in the final 2 hours. Plasma glucose levels in rats receiving saline, lipid, and CPT1A inhibitor during systemic lipid infusion (LI + CPT). The hyperglycemia induced by a physiologic increase in circulating LCFA levels in OF rats was prevented by the infusion of CPT1A inhibitor. (B) Total LCFA-CoA, linoleyl-CoA, and oleyl-CoA levels in the MBH obtained at the end of the pancreatic insulin clamp. (C) After 3 days' voluntary overfeeding, LCFAs failed to increase the MBH levels of LCFACoAs and to decrease food intake and liver glucose fluxes. The central inhibition of CPT1A restored the regulation of MBH LCFA-CoAs and normalized food intake and liver glucose fluxes. ${ }^{*} P<0.05$ CPT1A inhibitor versus control.

We as well as others have also focused on the initial phase in the development of diet-induced obesity and insulin resistance in rats in the hope of identifying early events that may be causative for the establishment of the phenotype $(20,21,34)$. These studies have revealed that when Sprague-Dawley rats are exposed to a highly palatable (lard-enriched) diet, they rapidly double their caloric intake, acquire severe hepatic insulin resistance, and fail to respond to the systemic administration of leptin (20) and the central administration of the LCFA oleic acid (21). The latter finding led us to the hypothesis that a rapid impairment in hypothalamic nutrient sensing plays an important role in the onset of obesity and insulin resistance in this model. To begin examining this postulate, we needed to address the following 2 critical questions:

Is the bypothalamic sensing of circulating lipids impaired following short-term overfeeding? We have recently shown that lipid infusion designed to double the circulating levels of LCFAs leads to a dou- bling of the levels of esterified LCFAs in the MBH (16). Furthermore, when the local esterification of LCFAs is pharmacologically inhibited, an increase in the availability of lipids fails to promote the accumulation of LCFA-CoAs within the MBH and leads to severe disruption of liver glucose homeostasis (16). Using a similar lipidinfusion protocol, here we demonstrate that doubling the circulating levels of LCFAs induced hyperglycemia and failed to increase the levels of LCFA-CoAs in the MBH of OF rats. This appears to be due to the increased utilization of LCFA-CoAs in the MBH mediated by an increase in the in vivo activity of CPT1, which is in turn mediated at least in part by decreased malonyl-Coa levels.

Is the restoration of hypothalamic lipid sensing sufficient to normalize energy and glucose homeostasis in this model? The above findings gave us an opportunity to test the role of defective sensing of lipids in the hypothalamus in the development of obesity and hepatic insulin resistance through a classic reconstitution experiment. Since 
pharmacologic or molecular inhibition of CPT1A activity in the hypothalamus can selectively increase MBH LCFA-CoA levels (18), we used these experimental approaches in order to elevate the levels of LCFA-CoAs in the MBH of OF rats. Indeed, curtailing the rate of oxidative lipid metabolism within the hypothalamus was sufficient to restore hypothalamic lipid sensing and to dramatically decrease food intake and glucose production in OF rats.

An important role of hypothalamic lipid metabolism in the regulation of energy homeostasis is also supported by numerous reports on the potent effects of inhibitors of fatty acid synthase (FAS) on body weight, food intake, and substrate oxidation $(11,13$, 35-38). While the precise mechanism by which systemic or central administration of the FAS inhibitor C75 leads to multiple effects on energy balance remains to be established, it is noteworthy that the anorectic effects of the systemic administration of C75 is preserved in genetic (39) and acquired rodent models of obesity (11, $35,36)$. Since the hypothalamic accumulation of malonyl-CoA, a potent inhibitor of CPT1 activity, has been implicated in the central effects of C75 (38), these findings appear to be consistent with the preserved effects of the direct pharmacologic or molecular inhibition of CPT1A inhibition in OF rats.

Our findings suggest that the compounded effects of a decrease in malonyl-CoA levels and an increase in CPT1 activity negate lipids' ability to increase the cellular levels of LCFA-CoAs in the $\mathrm{MBH}$. Most importantly, the observation that restoring the hypothalamic levels of esterified fatty acids to those observed in normal rats is sufficient to curtail both feeding behavior and glucose production indicates that hypothalamic lipid sensing plays a critical role in the maintenance of energy and glucose homeostasis. Since severe leptin resistance is also a feature of this model (20), one has to postulate that the MBH levels of LCFA-CoAs either lie downstream of the leptin signaling pathway (14) or can exert their anorexigenic and metabolic actions even in the presence of impaired leptin signaling.

In conclusion, this model of diet-induced obesity and insulin resistance displayed defective adaptation to an increase in lipid availability coupled with a severe impairment in the hypothalamic sensing of lipids. Importantly, restoration of hypothalamic lipid sensing by central inhibition of fatty acid oxidation was sufficient to normalize feeding behavior and glucose homeostasis. Further studies will be required to establish the critical role of this biochemical pathway in nutrient sensing in other animal models and in humans.

\section{Methods}

Animal preparation. We studied 147 ten-week-old male Sprague-Dawley rats (Charles River Laboratories). Rats were housed in individual cages and subjected to a standard light-dark cycle. Three weeks prior to the in vivo studies, rats underwent stereotaxic surgery to indwell a single catheter in the third cerebral ventricle $(10,40)$ or bilateral catheters into the $\mathrm{MBH}$ (41). One week later, indwelling catheters were placed in the internal jugular vein and carotid artery $(10,40)$. SC animals were fed standard chow (catalog no. 5001; Purina Mills Inc.), and OF animals were fed a highly palatable high-fat diet (catalog no. 9389; Purina Mills Inc.) that was generated by supplementing the SC diet with $10 \%$ lard (Supplemental Table 3). The study protocol was reviewed and approved by the Institutional Animal Care and Use Committee of the Albert Einstein College of Medicine.

Feeding behavior studies. This experimental protocol was designed to examine the acute effect of central inhibition of CPT1A on food intake in experimental groups SC and OF. SC and OF animals were allowed to eat their diets ad libitum. Rats were adapted to the metabolic cages, and their daily food intake had been constant (changed $<10 \%$ ) for a minimum of 3 consecutive days preceding the allocation to each group diet. Food intake was measured daily for 3 days after injection.

Pharmacologic inbibition of CPT1A. Following 3 days of ad libitum feeding (Figure 1A, day 0 ) and 3 hours before the onset of the dark cycle, rats received an icv bolus injection of either CPT1A inhibitor (5 and $25 \mathrm{pmol}$ ) or control compound (25 pmol) (Supplemental Figure 1).

Molecular inbibition of CPT1A. Following 2 days of ad libitum feeding (Figure $1 \mathrm{~A}$, day 0 ), rats received an icv bolus injection of CPT1A-Ribo or pTarget vector alone (Supplemental Figure 1) (18).

Conditioned taste aversion. Conditioned taste aversion was tested as described previously (42). Rats received icv CPT1 inhibitor (50 pmol/5 $\mu \mathrm{l})$, i.p. $\mathrm{LiCl}(22 \mathrm{mg} / \mathrm{kg})$ as the positive control, or icv artificial cerebrospinal fluid. Data are expressed as a preference ratio of saccharine intake to total fluid intake (i.e., saccharin plus water intake).

Pharmacologic inhibition of CPT1A. At $t=0$ minutes, we began a continuous icv infusion of either a reversible CPT1A inhibitor or its inactive stereoisomer at a rate of $50 \mathrm{pmol} / \mathrm{h}$ in a volume of $5 \mu \mathrm{l} / \mathrm{h}$ (Supplemental Figure 1C), and maintained it throughout the study (6 hours). For the pancreatic clamp performed during lipid infusion, the CPT1A inhibitor or the control compound ( $1 \mathrm{mM}$ solution) were delivered in the $\mathrm{MBH}$ for 6 hours at a rate of $0.33 \mu \mathrm{l} / \mathrm{h}$. CPT1A inhibitor and control compound were dissolved in artificial cerebrospinal fluid (Harvard Apparatus).

Molecular inbibition of CPT1A. Two days before the pancreatic insulin clamp, rats received the icv injection of CPT1A-Ribo or vector control. Controls were pair-fed with the rats receiving CPT1A-Ribo.

Clamp studies. At 120 minutes, a primed continuous infusion of [3- ${ }^{3} \mathrm{H}$ ]-glucose ( $40 \mu \mathrm{Ci}$ bolus, $0.4 \mu \mathrm{Ci} / \mathrm{min}$; New England Nuclear) purified by high-performance liquid chromatography (HPLC) was started and continued for the duration of the study $(15,18,24)$. Samples for determination of $\left[3-{ }^{3} \mathrm{H}\right]$-glucose-specific activity were obtained at 10 -minute intervals. Finally, a pancreatic insulin clamp $(15,18,24)$ was initiated at 240 minutes and lasted for 2 hours. This procedure involved the infusion of somatosta$\operatorname{tin}(3 \mu \mathrm{g} / \mathrm{kg} / \mathrm{min})$, insulin $(1 \mathrm{mU} / \mathrm{kg} / \mathrm{min})$. Additionally, a variable infusion of a $25 \%$ glucose solution was started and periodically adjusted to clamp the plasma glucose concentration at approximately $8 \mathrm{mM}$.

Lipid infusion studies. At 120 minutes, rats received i.v. saline or $20 \%$ intralipid (mixed with $20 \mathrm{U} / \mathrm{ml}$ of heparin, $0.4 \mathrm{ml} / \mathrm{h}$ ) and $\left[3-{ }^{3} \mathrm{H}\right]$-glucose for 4 hours (16). A pancreatic clamp was performed in the final 2 hours as described above. Intralipid was purchased from Baxter and consisted of $26 \%$ oleic acid (18:1), 50\% linoleic acid (18:2), 9\% linolenic acid (18:3), 10\% palmitic acid (16:0), and 3.5\% stearic acid (18:0).

Analytical procedures. Plasma samples for determination of plasma free fatty acid, insulin, and leptin concentrations were obtained at 30-minute intervals during the study. Ten minutes before the end of the studies, [U-14 $\mathrm{C}]$-lactate (20 $\mu \mathrm{Ci}$ bolus; $1.0 \mu \mathrm{Ci} / \mathrm{min}$; New England Nuclear) was administered to determine the contribution of gluconeogenesis to the pool of hepatic G6P (43). At the end of the clamp studies rats were anesthetized, and tissue samples were freeze-clamped in situ with aluminum tongs pre-cooled in liquid nitrogen. All tissue samples were stored at $-800^{\circ} \mathrm{C}$ for subsequent analysis.

CPT1A-Ribo plasmid. CPT1A-Ribo plasmid (Supplemental Figure 1B) was prepared and delivered as previously described (18). Briefly, endotoxin-free plasmid DNA was complexed with polyethylenimine (Sigma-Aldrich) (44) and injected icv as a single bolus. Control studies used pTarget (Stratagene) plasmid DNA (Vector Laboratories).

LCFA-CoA measurements. LCFA-CoAs were extracted from mediobasal hypothalamic wedges and measured by HPLC as previously described (45).

$\mathrm{MBH}$ malonyl-CoA. The MBH was sampled, and malonyl-CoA was extracted and measured by a modification of an HPLC method previously described (46). 
Western blot analysis. Western blot analysis of wedge tissue (6-8 $\mathrm{mg})$ obtained from SC and OF rats was performed as described previously (47). Membranes were immunoblotted with ACC, pACC, AMPK, pAMPK, and FAS primary antibodies (Cell Signaling Technology). Immune complexes were detected by enhanced chemiluminescense (Western Lightning; PerkinElmer) using horseradish peroxidase-conjugated secondary antibodies. Bands were imaged on X-ray film and digitized for quantification using ImageQuant software (Molecular Dynamics Inc.). $\beta$-Tubulin was used for loading normalization.

CPT1 activity assay. Arcuate nuclei obtained by micropunch technique (18) were homogenized in $50 \mu \mathrm{l}$ buffer containing $0.25 \mathrm{M}$ sucrose, $1 \mathrm{mM}$ EDTA, and $10 \mathrm{mM}$ Tris- $\mathrm{HCl}, \mathrm{pH}$ 7.4. The homogenate was fractionated by centrifugation at $20,000 \mathrm{~g}$ for 20 minutes. CPT enzymatic activity was measured as described previously $(18,48)$. The results are expressed as percentage of control CPT1 specific activity (i.e. nmol palmitoyl-CoA produced per min per mg of protein).

Acyl-CoA bydrolase activity assay. Rat arcuate extracts (approximately $5 \mu \mathrm{g}$ of protein) were incubated in the presence of $3.7 \mathrm{kBq}(66 \mu \mathrm{M})$ of $\left[{ }^{14} \mathrm{C}\right]$-palmitoyl-CoA at $37^{\circ} \mathrm{C}$ in $25 \mu \mathrm{l}$ of reaction buffer $(50 \mathrm{mM}$ Tris- $\mathrm{HCl}$, $\mathrm{pH} 7.0,1 \mathrm{mM} \mathrm{CaCl}_{2}$, BSA $1 \mathrm{mg} / \mathrm{ml}$ ). After 1 hour incubation the reactions were terminated by chilling on ice. Free $\left[{ }^{14} \mathrm{C}\right]$-palmitate was extracted with $\mathrm{N}$-hexane and measured by scintillation counting. Activity is expressed as cpm of $\left[{ }^{14} \mathrm{C}\right]$-palmitate/ $\mu \mathrm{g}$ protein.

Quantitation of CPT1A and CPT1B mRNA. Total RNA was isolated with Trizol (Invitrogen Corp.) from individual hypothalamic nuclei. Singlestranded cDNA synthesis, and quantitative expression for CPT1A and $C P T 1 B$ was measured by quantitative RT-PCR as described previously (18). The copy number of each transcript was measured against a copy number standard curve of cloned target templates. Expression of each transcript was normalized to the copy number for $\beta$-actin.

G6pc and Pck1 expression. Liver G6pc and phosphoenolpyruvate carboxykinase (Pck1) mRNA abundance were assessed by quantitative real-time PCR as previously described $(16,24)$. The copy number of each transcript was derived from a standard curve of cloned target templates. Expression of each transcript was normalized to the copy number for Gapdh.

Brain stereotactic micropunches and $M B H$ wedge. Brain micropunches of individual hypothalamic nuclei were prepared as previously described (18), and the $\mathrm{MBH}$ was sampled by dissecting a wedge of tissue including the entire mediolateral and dorsoventral extent of the arcuate nuclei while minimizing ventromedial nucleus tissue (16).

Hepatic glucose fluxes. The rates of hepatic glucose fluxes were determined as previously described (43).

Statistics. All values are presented as means \pm SEM. Comparisons among groups were made using ANOVA or unpaired Student's $t$ tests, as appropriate.

\section{Acknowledgments}

We wish to thank B. Liu, Y. Wang, C. Baveghems, and S. Gaweda for expert technical assistance. This work was supported by grants from the National Institutes of Health (DK 48321, DK 45024, and AG 21654 to L. Rossetti), the AECOM Diabetes Research \& Training Center (DK 20541), and the Skirball Foundation. S. Obici is the recipient of a Junior Faculty Award from the American Diabetes Association. T.K.T. Lam is supported by a Training Grant from the National Institute on Aging (T32-AG023475). A. Pocai and E.D. Muse are supported by the American Diabetes Association.

Received for publication August 19, 2005, and accepted in revised form January 17, 2006.

Address correspondence to: Luciano Rossetti, Diabetes Research Center, Albert Einstein College of Medicine, 1300 Morris Park Avenue, New York, New York 10461, USA. Phone: (718) 430-4118; Fax: (718) 430-8557; E-mail: rossetti@aecom.yu.edu.
1. Flegal, K.M., Carroll, M.D., Ogden, C.L., and Johnson, C.L. 2002. Prevalence and trends in obesity among US adults, 1999-2000. JAMA. 288:1723-1727.

2. Finer, N. 2003. Obesity. Clin. Med. 3:23-27.

3. Obici, S., Feng, Z., Karkanias, G., Baskin, D.G., and Rossetti, L. 2002. Decreasing hypothalamic insulin receptors causes hyperphagia and insulin resistance in rats. Nat. Neurosci. 5:566-572.

4. Woods, S.C., Lotter, E.C., McKay, L.D., and Porte, D., Jr. 1979. Chronic intracerebroventricular infusion of insulin reduces food intake and body weight of baboons. Nature. 282:503-505.

5. Brief, D.J., and Davis, J.D. 1984. Reduction of food intake and body weight by chronic intraventricular insulin infusion. Brain Res. Bull. 12:571-575.

6. Schwartz, M.W., Seeley, R.J., Campfield, L.A., Burn, P., and Baskin, D.G. 1996. Identification of targets of leptin action in rat hypothalamus. J. Clin. Invest. 98:1101-1106.

7. Schwartz, M.W., et al. 1996. Specificity of leptin action on elevated blood glucose levels and hypothalamic neuropeptide $Y$ gene expression in ob/ob mice. Diabetes. 45:531-535.

8. Frederich, R.C., et al. 1995. Leptin levels reflect body lipid content in mice: evidence for diet-induced resistance to leptin action. Nat. Med. 1:1311-1314.

9. Zhang, Y., et al. 1994. Positional cloning of the mouse obese gene and its human homologue. Nature. 372:425-432.

10. Obici, S., et al. 2002. Central administration of oleic acid inhibits glucose production and food intake. Diabetes. 51:271-275.

11. Shimokawa, T., Kumar, M.V., and Lane, M.D. 2002. Effect of a fatty acid synthase inhibitor on food intake and expression of hypothalamic neuropeptides. Proc. Natl. Acad. Sci. U. S. A. 99:66-71.

12. Makimura, H., et al. 2001. Cerulenin mimics effects of leptin on metabolic rate, food intake, and body weight independent of the melanocortin system, but unlike leptin, cerulenin fails to block neuroendocrine effects of fasting. Diabetes. 50:733-739.

13. Loftus, T.M., et al. 2000. Reduced food intake and body weight in mice treated with fatty acid synthase inhibitors. Science. 288:2379-2381.

14. Minokoshi, Y., et al. 2004. AMP-kinase regulates food intake by responding to hormonal and nutrient signals in the hypothalamus. Nature. 428:569-574.

15. Pocai, A., Obici, S., Schwartz, G.J., and Rossetti, L. 2005. A brain-liver circuit regulates glucose homeostasis. Cell Metab. 1:53-61.

16. Lam, T.K., et al. 2005. Hypothalamic sensing of circulating fatty acids is required for glucose homeostasis. Nat. Med. 11:320-327.

17. Schwartz, M.W., and Porte, D., Jr. 2005. Diabetes, obesity, and the brain. Science. 307:375-379.

18. Obici, S., Feng, Z., Arduini, A., Conti, R., and Rossetti, L. 2003. Inhibition of hypothalamic carnitine palmitoyltransferase-1 decreases food intake and glucose production. Nat. Med. 9:756-761.

19. Kraegen, E.W., et al. 1991. Development of muscle insulin resistance after liver insulin resistance in high-fat-fed rats. Diabetes. 40:1397-1403.

20. Wang, J., et al. 2001. Overfeeding rapidly induces leptin and insulin resistance. Diabetes. 50:2786-2791.

21. Morgan, K., Obici, S., and Rossetti, L. 2004. Hypothalamic responses to long-chain fatty acids are nutritionally regulated. J. Biol. Chem. 279:31139-31148.

22. Bjorbak, C., et al. 2000. SOCS3 mediates feedback inhibition of the leptin receptor via Tyr985. J. Biol. Chem. 275:40649-40657.

23. Munzberg, H., and Myers, M.G., Jr. 2005. Molecular and anatomical determinants of central leptin resistance. Nat. Neurosci. 8:566-570.
24. Pocai, A., et al. 2005. Hypothalamic K(ATP) channels control hepatic glucose production. Nature. 434:1026-1031.

25. Flier,J.S. 2004. Obesity wars: molecular progress confronts an expanding epidemic. Cell. 116:337-350.

26. Taylor, S.I. 1999. Deconstructing type 2 diabetes. Cell. 97:9-12.

27. Friedman, J.M. 2003. A war on obesity, not the obese. Science. 299:856-858.

28. Obici, S., and Rossetti, L. 2003. Nutrient sensing and the regulation of insulin action and energy balance. Endocrinology. 144:5172-5178.

29. Mobbs, C.V., and Makimura, H. 2002. Block the FAS, lose the fat. Nat. Med. 8:335-336.

30. El Haschimi, K., Pierroz, D.D., Hileman, S.M., Bjorbaek, C., and Flier, J.S. 2000. Two defects contribute to hypothalamic leptin resistance in mice with diet-induced obesity. J. Clin. Invest. 105:1827-1832.

31. Munzberg, H., Flier, J.S., and Bjorbaek, C. 2004. Region-specific leptin resistance within the hypothalamus of diet-induced obese mice. Endocrinology. 145:4880-4889.

32. Van Heek, M., et al. 1997. Diet-induced obese mice develop peripheral, but not central, resistance to leptin. J. Clin. Invest. 99:385-390.

33. Clegg, D.J., et al. 2005. Reduced anorexic effects of insulin in obesity-prone rats fed a moderatefat diet. Am. J. Physiol. Regul. Integr. Comp. Physiol. 288:R981-R986.

34. Storlien, L.H., James, D.E., Burleigh, K.M., Chisholm, D.J., and Kraegen, E.W. 1986. Fat feeding causes widespread in vivo insulin resistance, decreased energy expenditure, and obesity in rats. Am. J. Physiol. 251:E576-E583.

35. Kumar, M.V., Shimokawa, T., Nagy, T.R., and Lane, M.D. 2002. Differential effects of a centrally acting fatty acid synthase inhibitor in lean and obese 
mice. Proc. Natl. Acad. Sci. U. S. A. 99:1921-1925.

36. Thupari, J.N., Landree, L.E., Ronnett, G.V., and Kuhajda, F.P. 2002. C75 increases peripheral energy utilization and fatty acid oxidation in diet-induced obesity. Proc. Natl. Acad. Sci. U. S. A. 99:9498-9502.

37. Kim, E.K., et al. 2002. Expression of FAS within hypothalamic neurons: a model for decreased food intake after C75 treatment. Am. J. Physiol. Endocrinol. Metab. 283:E867-E879.

38. Kim, E.K., et al. 2004. C75, a fatty acid synthase inhibitor, reduces food intake via hypothalamic AMP-activated protein kinase. J. Biol. Chem. 279:19970-19976.

39. Cha, S.H., Hu, Z., and Lane, M.D. 2004. Long-term effects of a fatty acid synthase inhibitor on obese mice: food intake, hypothalamic neuropeptides, and UCP3. Biochem. Biophys. Res. Commun. 317:301-308.

40. Obici, S., Zhang, B.B., Karkanias, G., and Rossetti,
L. 2002. Hypothalamic insulin signaling is required for inhibition of glucose production. Nat. Med. 8:1376-1382.

41. Morton, G.J., et al. 2003. Arcuate nucleus-specific leptin receptor gene therapy attenuates the obesity phenotype of Koletsky $(\mathrm{fa}(\mathrm{k}) / \mathrm{fa}(\mathrm{k}))$ rats. Endocrinology. 144:2016-2024.

42. Benoit, S.C., et al. 2003. Assessment of the aversive consequences of acute and chronic administration of the melanocortin agonist, MTII. Int. J. Obes. Relat. Metab. Disord. 27:550-556.

43. Barzilai, N., et al. 1997. Leptin selectively decreases visceral adiposity and enhances insulin action. J. Clin. Invest. 100:3105-3110.

44. Boussif, O., et al. 1995. A versatile vector for gene and oligonucleotide transfer into cells in culture and in vivo: polyethylenimine. Proc. Natl. Acad. Sci.
U. S. A. 92:7297-7301.

45. Woldegiorgis, G., Spennetta, T., Corkey, B.E., Williamson, J.R., and Shrago, E. 1985. Extraction of tissue long-chain acyl-CoA esters and measurement by reverse-phase high-performance liquid chromatography. Anal. Biochem. 150:8-12.

46. Hosokawa, Y., Shimomura, Y., Harris, R.A., and Ozawa, T. 1986. Determination of short-chain acyl-coenzyme A esters by high-performance liquid chromatography. Anal. Biochem. 153:45-49.

47. Pocai, A., et al. 2005. Central leptin acutely reverses diet-induced hepatic insulin resistance. Diabetes. 54:3182-3189.

48. Arduini, A., et al. 1994. Evidence for the involvement of carnitine-dependent long-chain acyltransferases in neuronal triglyceride and phospholipid fatty acid turnover. J. Neurochem. 62:1530-1538. 